\title{
Supportive Care in Head and Neck Cancers: Multidisciplinary Management
}

\author{
Presented by Jimmy J. Caudell, MD, PhD; David G. Pfister, MD; and Randal S. Weber, MD
}

\begin{abstract}
Patients with head and neck cancer experience a broad array of negative quality-of-life issues, but particularly common are dental complications following radiation therapy, and compromised nutrition with significant weight loss. At the NCCN 2021 Virtual Annual Conference, a panel of experts used a case-based approach to discuss some of these common adverse effects of head and neck cancer and its treatment, as well as optimal supportive care strategies to manage them.

\begin{abstract}
Although a broad spectrum of quality-of-life issues impact patients with head and neck cancers (HNC), a few are paramount: receiving inadequate nutrition and having dental complications after radiation therapy. In a panel moderated by David G. Pfister, MD, Chair of the NCCN Head and Neck Cancers Panel, at the NCCN 2021 Virtual Annual Conference, Dr. Pfister, along with Jimmy J. Caudell, MD, PhD, and Randal S. Weber, MD, both members of the NCCN HNC Panel, used a casebased approach to discuss optimal supportive care strategies for these particularly impactful quality-of-life issues in patients with HNC.
\end{abstract}

\section{Epidemiology}

Potential primary sites of HNC include the oral cavity, pharynx, larynx, nasal cavity, and paranasal sinuses. In 2019, more than 65,000 new cases of HNC were diagnosed, and there were more than 14,000 deaths due to the disease. Patients with these malignancies have a median age at diagnosis of 60 years and are predominantly men. Further, the mortality incidence is greatest among African Americans compared with other ethnic groups. ${ }^{1}$

"Historically, HNC diagnosis has been strongly associated with tobacco and alcohol, but for a while we have appreciated the role of Epstein-Barr virus (EBV) as a risk factor for nasopharynx cancer," noted Dr. Pfister, Chief, Head and Neck Oncology Service, and Co-Leader, MultiDisciplinary Head and Neck Cancer Disease Management Team, Memorial Sloan Kettering Cancer Center. "But, more recently, what is receiving a lot of headlines is human papillomavirus [HPV] infection. It's commonly seen in the lay press, but it's important to note that we cure most patients with HPV-related disease, even at advanced stages." Patients with HPV-positive HNC often present at a younger age, so they live with the adverse effects (AEs) of treatment for a longer time than their older counterparts, he added.

Most patients with HNC present with locoregionally advanced disease, and require a combined modality approach to treatment (ie, surgery and radiation, chemotherapy and radiation, or even all 3 modalities). These patients also tend to have significant treatment-related AEs. According to Dr. Pfister, managing these AEs requires a team approach, and supportive care responsibilities apply to many members of the multidisciplinary team, outlined in the NCCN Clinical Practice Guidelines in Oncology (NCCN Guidelines) for Head and Neck Cancers.

"Patients can sometimes bounce around from provider to provider without knowing who really owns their [supportive care] management," he said. "I think we really need to embrace the team approach with HNC in general, but particularly in the supportive care setting."

\section{HPV-Positive Squamous Carcinoma of the Oropharynx}

Dr. Weber, Professor, Department of Head and Neck Surgery, and adjunct Professor, Department of Radiation Oncology, The University of Texas MD Anderson Cancer Center, and Adjunct Professor, Department of Otolaryngology, Head and Neck Surgery, Baylor College of Medicine, presented the case of a 54-year-old man presenting to his primary care physician with a sore throat and mass in his neck. He was then referred to an ENT, who observed a large mass in the vallecula and epiglottis. The patient then underwent an endoscopy with a biopsy of the mass, but suffered an airway obstruction that warranted an emergency tracheostomy. He was then referred to MD Anderson Cancer Center, where he was diagnosed 
with stage T2N1 cancer. Pretreatment therapy consisted of a modified barium swallow (MBS), and his swallowing returned to normal.

Dr. Weber noted that his patients always receive an MBS score at baseline from a speech pathologist. "Swallowing function pretreatment can predict posttreatment function," he noted. "So it's an important baseline, and therefore we integrate our speech and language pathologists throughout the treatment course" (Figure 1).

According to Dr. Weber, the patient's tumor was debulked, so the volume of disease was not as extensive when he underwent the endoscopy. The primary tumor involved both the epiglottis and tongue base, with 2 PETpositive nodes on the left of his neck. Recommendations from the multidisciplinary tumor board included 70 Gy of intensity-modulated radiation therapy (IMRT) in 200-cGy fractions using a supraglottic larynx volumetric modulated arc therapy protocol, along with concurrent cisplatin, $40 \mathrm{mg} / \mathrm{m}^{2}$ weekly. Pretreatment, the patient had a body mass index of $32 \mathrm{~kg} / \mathrm{m}^{2}$, with normal swallowing function. To ensure adequate nutritional status throughout concurrent therapy, Dr. Weber said it's important to maintain active swallowing therapy and to place a percutaneous endoscopic gastrostomy (PEG) tube when $10 \%$ of body weight has been lost (Figure 3 ).

"The trigger to place a PEG tube is when the patient has lost $10 \%$ of their ideal body weight, when we really have to prevent further weight loss, optimize nutrition, and maintain hydration," he said. "This will decrease emergency room visits and what we want to avoid at all costs: treatment breaks. Continued swallowing on the part of the patient is important; if you don't use it, you lose it."

The patient experienced severe mucositis but completed the prescribed dose of radiation on time. He had poor oral intake and weight loss, and at the fourth week posttreatment he met criteria for placement of a PEG tube. With continued therapy, his dysphagia improved initially, but progressively worsened. "At 6 months posttreatment, he was really struggling," said Dr. Weber. The patient's follow-up MBS score demonstrated complete pharyngeal stenosis, but a review of the treatment plan and doses delivered did not identify any excessive or unplanned dose to the inferior constrictor or cricopharyngeal muscle. "Everything done was appropriate," he noted. Based on these factors, Dr. Weber said the best approach was determined to be an esophageal dilatation with a rendezvous technique to reestablish the esophageal lumen. Through aggressive dilatation, the patient was able to reestablish swallowing function.

Discussing this case, Dr. Weber noted that prevention of pharyngeal stenosis is crucial, and that continued swallowing can diminish the risk of stenosis. This highlights the importance of measuring swallowing function before, during, and after radiation, and continuing swallowing therapy throughout treatment. "Frequently patients will need a PEG tube, particularly when they're getting multimodality treatment; the trigger is typically a weight loss of $10 \%$ or multiple emergency room visits requiring hydration," he said. "But that gets expensive and it's disruptive to the patient's life."

Pharyngeal stenosis is more easily managed if caught early, when there is still a chance for dilatation. The rendezvous technique has been proven to be beneficial and safe, but in extreme cases, extensive surgery with loss of the larynx and reconstruction of the pharynx is necessary. "But, we typically reserve that for patients who have had multiple episodes of aspiration," he noted.

\section{Xerostomia/Dry Mouth}

Dr. Pfister presented the case of a man aged 65 years with HPV-negative, T2N2bM0 cancer at the tongue base. He had ipsilateral disease, with 2 positive nodes in the upper neck, both measuring $<3 \mathrm{~cm}$. It was recommended that he initiate treatment with chemoradiotherapy.

"This is a patient with a prognosis that is probably not as good as a patient with HPV-positive disease," he said. According to Dr. Pfister, this patient was aware of dry mouth as an $\mathrm{AE}$ of radiation and wanted to learn more about interventions that might mitigate its severity. Dry mouth is among the most common long-term effects of HNC radiation, and is a major adverse quality-of-life factor for these patients. Its impact and severity is related to dose and target/primary site, as well as the extent of disease. Saliva is critical to oral health, and chronic dry mouth contributes to oral infection, cavities, periodontal disease, oral discomfort, and taste alteration; therefore, it has an understandably profound impact on nutrition.

“But if you're proactive about [dry mouth] and you mitigate it, you can definitely improve the quality of life of these patients," he said, noting that he uses a 2-pronged approach: first preventing it, then mitigating it once it occurs. According to the literature, some strategies to mitigate dry mouth include newer conformal radiation techniques (ie, IMRT, proton therapy) that spare salivary tissue, ${ }^{2}$ submandibular gland transfer (in selected patients), ${ }^{3}$ amifostine (although associated with its own AEs and can be logistically complicated), ${ }^{4}$ and bethanechol (may lead to worse dry mouth when discontinued). ${ }^{5}$

In patients with established dry mouth, other mitigation techniques can be helpful, such as topical measures (ie, artificial saliva, humidity, increased water intake), modified diet and lifestyle, sugarless candies and gums that generate extra saliva, cholinomimetic agents that increase saliva (although often associated with off-target effects like sweating and headache), ${ }^{6}$ acupuncture, ${ }^{7}$ and hyperbaric oxygen (requires more confirmation). ${ }^{8}$ 


\section{PRINCIPLES OF NUTRITION: MANAGEMENT AND SUPPORTIVE CARE ${ }^{1-3}$}

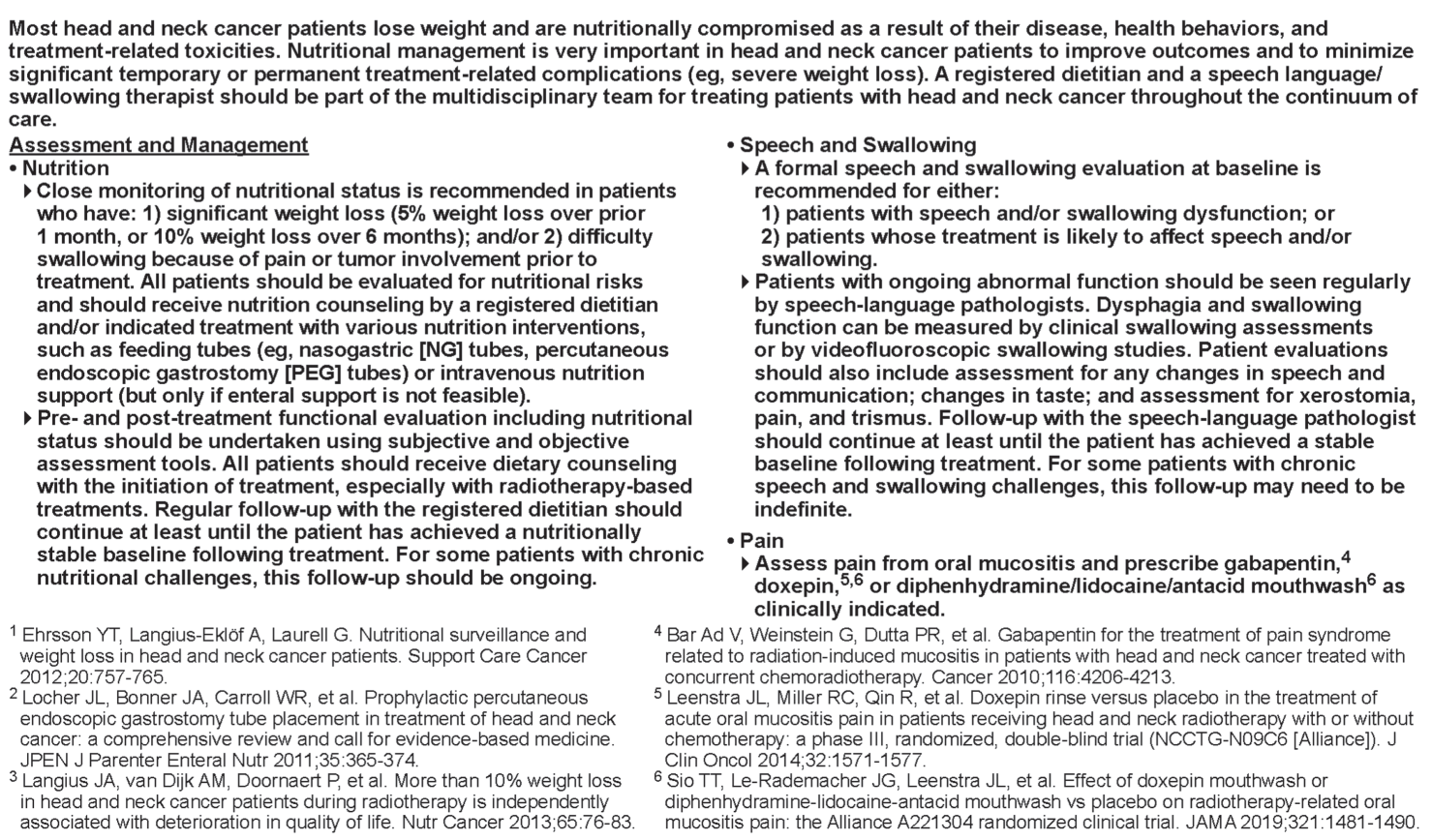

Figure 1. Principles of nutrition: management and supportive care (NUTR-A 1 of 2). From the NCCN Guidelines for Head and Neck Cancers. Version 1.2021. To view the most recent version, visit NCCN.org.

()2021 National Comprehensive Cancer Network ${ }^{\circledR}\left(\mathrm{NCCN}^{\circledR}\right)$, All rights reserved. This illustration may not be reproduced in any form without the express written permission of NCCN.

\section{EBV-Positive Nasopharyngeal Cancer}

Dr. Caudell, Associate Member, and Vice-Chair, Clinical Research, Department of Radiation Oncology, Moffitt Cancer Center, discussed the case of a 61-year-old-man who presented in 2014 with sinus congestion, a 15-pound weight loss, and decreased hearing in his left ear. Upon examination, he was diagnosed with T3N3bM0 EBV-positive nasopharyngeal carcinoma. Radiation treatment was initiated with $69.3 \mathrm{~Gy}$ to gross disease and 54.12 Gy to the elective neck, all in 33 fractions. He also received concurrent cisplatin, $100 \mathrm{mg} / \mathrm{m}^{2}$.

"We were able to spare his parotids very effectively. However, if you look in the axial slice, you'll see his submandibular glands are right next to the high-dose volume radiation, so we weren't able to spare those nearly as well," he noted. The patient required hospitalization during treatment, secondary to febrile neutropenia. He received empiric antibiotics and antifungal medications, the neutropenia and fever resolved, and he was discharged. He did not require a nasal gastric tube or a PEG tube during treatment, and he went on to receive adjuvant chemotherapy (as was the standard at the time) with carboplatin and paclitaxel for 2 cycles.

According to Dr. Caudell, this patient did well for years, but in 2019 presented with perceived slurring of speech. On examination, his tongue deviated to the right, suggestive of a hypoglossal nerve palsy. An MRI was performed and was negative for disease, suggesting that radiation neuropathy was the cause of the patient's slurred speech. For this type of patient, the risk of a lower cranial neuropathy, or more specifically a hypoglossal neuropathy, at 5 years posttreatment is approximately $2 \%$, he said. ${ }^{9}$

He noted a recent study out of MD Anderson that looked at the risk of lower cranial neuropathy in almost 2,000 patients with oropharyngeal cancer. ${ }^{10}$ "Their numbers at 5 years pretty much agree-at around $2 \%-$ although this risk increases with even longer follow-up," he added. The study also suggested some other risk factors for late cranial neuropathy, including accelerated radiation and neurotoxic chemotherapy agents.

"This particular patient did receive accelerated radiation in addition to adjuvant paclitaxel, which may have impacted his risk," he added.

According to Dr. Caudell, supportive care for patients with EBV-positive nasopharyngeal cancer should take into account the risk of hospitalization during therapy and the risk of lower cranial neuropathy after radiation therapy, as well as proper management of neck stiffness/ neck muscle spasms. "Currently, there's not a whole lot that I'm aware of to treat neuropathies posttreatment," he said. "There is some evidence that bevacizumab or nerve growth factor may potentially be helpful in patients 
with temporal lobe necrosis, but this has not been tested specifically in neuropathy."

"Cisplatin and paclitaxel are certainly neurotoxic, but my sense is that the big offender here is probably related to radiation," Dr. Pfister added. "Once patients have cranial neuropathy, I generally find that it's more of a rehabilitation scenario than it is a reversal. But gabapentin can certainly decrease some of the neuropathic pain. However, I think prevention is really the key." Dr. Weber added that most of the literature on the topic is anecdotal, and that clinical trials are needed in this space.
Disclosures: Dr. Caudell has disclosed receiving grant/research support from Varian Medical Systems, Inc. Dr. Pfister has disclosed receiving grant/research support from Atara Biotherapeutics and Merck \& Co., Inc. Dr. Weber has disclosed no relevant financial relationships.

Correspondence: Jimmy J. Caudell, MD, PhD, Moffitt Cancer Center, Department of Radiation Oncology, 12902 Magnolia Drive, Tampa, FL 33612. Email: jimmy.caudell@moffitt.org;

David G. Pfister, MD, Memorial Sloan Kettering Cancer Center, 530 East 74th Street, New York, NY 10021. Email: pfisterd@mskcc.org; and

Randal S. Weber, MD, The University of Texas MD Anderson Cancer Center, Department of Head and Neck Surgery, 1400 Pressler Street, Unit 1445, Houston, TX 77030. Email: rsweber@mdanderson.org

\section{References}

1. Siegel RL, Miller KD, Jemal A, et al. Cancer statistics, 2019. CA Cancer J Clin 2019;69:7-34

2. Nutting $\mathrm{CM}$, Morden JP, Harrington $\mathrm{KJ}$, et al. Parotid-sparing intensity modulated versus conventional radiotherapy in head and neck cancer (PARSPORT): a phase 3 multicentre randomised controlled trial. Lancet Oncol 2011;12:127-136.

3. Jha N, Harris J, Seikaly H, et al. A phase II study of submandibular gland transfer prior to radiation for prevention of radiation-induced xerostomia in head-and-neck cancer (RTOG 0244). Int J Radiat Oncol Biol Phys 2012;84:437-442.

4. Brizel DM, Wasserman TH, Henke $M$, et al. Phase III randomized trial of amifostine as a radioprotector in head and neck cancer. J Clin Oncol 2000;18:3339-3345.

5. Jaguar GC, Lima ENP, Kowalski LP, et al. Double blind randomized prospective trial of bethanechol in the prevention of radiation-induced salivary gland dysfunction in head and neck cancer patients. Radiother Oncol 2015;115:253-256.
6. Chambers MS, Posner M, Jones CU, et al. Cevimeline for the treatment of postirradiation xerostomia in patients with head and neck cancer. Int J Radiat Oncol Biol Phys 2007;68:1102-1109.

7. Pfister DG, Cassileth BR, Deng GE, et al. Acupuncture for pain and dysfunction after neck dissection: results of a randomized controlled trial. J Clin Oncol 2010;28:2565-2670.

8. Forner L, Hyldegaard O, von Brockdorff AS, et al. Does hyperbaric oxygen treatment have the potential to increase salivary flow rate and reduce xerostomia in previously irradiated head and neck cancer patients? A pilot study. Oral Oncol 2011;47:546-551.

9. Chow JCH, Cheung $\mathrm{K}, \mathrm{Au} \mathrm{K}$, et al. Radiation-induced hypoglossal nerve palsy after definitive radiotherapy for nasopharyngeal carcinoma: clinical predictors and dose-toxicity relationship. Radiother Oncol 2019;138:93-98.

10. Aggarwal P, Goepfert R, Garden AS, et al. Risk and clinical risk factors associated with late lower cranial neuropathy in long-term oropharyngeal squamous cell carcinoma survivors [published online February 4, 2021] JAMA Otolaryngol Head Neck Surg, doi: 10.1001/jamaoto.2020.5269 